\title{
3'-Sulfated Lewis A is a Biomarker for Metaplastic and Oncogenic Transformation of Several Gastrointestinal Epithelia
}

\author{
Jeffrey W. Brown, M.D., Ph.D. ${ }^{1 \dagger}$ \\ Koushik K. Das, M.D. ${ }^{1}$ \\ Vasilios Kalas, M.D., Ph.D. 2,3 \\ Kiron M. Das, M.D., Ph.D. ${ }^{4}$ \\ Jason C. Mills, M.D., Ph.D. ${ }^{1,5,6 \dagger}$
}

${ }^{1}$ Division of Gastroenterology, Department of Medicine, Washington University in St. Louis, School of Medicine, St. Louis, MO

${ }^{2}$ Washington University in St. Louis, School of Medicine, St. Louis, MO

${ }^{3}$ Physician Scientist Training Program, Department of Medicine, McGaw Medical Center of Northwestern University, Chicago, IL

${ }^{4}$ Division of Gastroenterology, Rutgers-Robert Wood Johnson Medical School, New Brunswick, New Jersey

${ }^{5}$ Department of Pathology and Immunology, Washington University in St. Louis, School of Medicine, St. Louis, MO

${ }^{6}$ Department of Developmental Biology, Washington University in St. Louis, School of Medicine, St. Louis, MO

†Correspondence should be addressed to:

Jeffrey W. Brown, M.D., Ph.D.

Washington University in St. Louis, School of Medicine

660 S. Euclid Avenue

Campus Box 8124

St. Louis, MO 63110

Email: brownjw@wustl.edu

or

Jason C. Mills, M.D., Ph.D.

Washington University in St. Louis, School of Medicine

660 S. Euclid Avenue

Campus Box 8124

St. Louis, MO 63110

Email:jmills@wustl.edu

Short Title: 3'-Sulfo-Le ${ }^{\mathrm{A}}$ in metaplasia and cancer

Keywords: Sulfation, Galactose, Barrett's Esophagus, Gastric Metaplasia, Gastric Cancer, Pan-

IN, Pancreatic Cancer 
Abbreviations used in this paper: BSA: Bovine Serum Albumin; ELISA: Enzyme Linked Immunosorbent Assay; Gal: Galactose; GIcNAc: N-Acetylglucosamine; PBS: Phosphate Buffered Saline, Gal: Galactose, Le ${ }^{A}$ : Lewis A, Le ${ }^{X}$ : Lewis X; TFMS: Trifluoromethanesulfonic Acid

Disclosures / Conflicts of Interest: KMD and KKD have been granted a patent for the use of Das1 in the detection of cancerous pancreatic lesions. KKD is providing Das-1 antibody to Interpace Biosciences for commercial use in risk stratifying pancreatic cystic lesions. JWB, VK, and JCM have no conflicts of interest.

Preprint Server: Not applicable

Glycan Profiling: www.functionalglycomics.org

Author Contributions: JWB, KKD, VK, KMD, and JCM conceived of the idea. JWB, VK performed the experiments. JWB, KKD, VK, and JCM analyzed the data. JWB, KKD, VK, KMD, and JCM prepared the manuscript. JWB, KKD, KMD, and JCM funded the research.

\section{Grant Support}

JWB: Department of Defense W81XWH-20-1-0630, NIH T32 DK007130-42, the Digestive Disease Research Core Centers Pilot and Feasibility Grant as part of P30 DK052574, and the American Gastroenterological Association AGA2021-5101.

JCM: R21DK111369, R01DK094989, R01DK105129, R01DK110406, P30 CA09182, R01 CA239645, R01 CA246208, the Alvin J. Siteman Cancer Center-Barnes Jewish Foundation Cancer Frontier Fund, and the BETRNet (U54CA163060).

V.K.: T32 GM07200

KMD: R01 DK47673, R01 DK63618

Other: The National Center for Functional Glycomics Glycan Array resource and much appreciated assistance with the analysis was supported by R24 GM098791, R24 GM137763, and P41 GM103694.

Word Count: 1591

Figure Count: 4

Table: 1

Supplemental Dataset: 3 (Large datasets)

Reference Count: 39 


\section{ABSTRACT}

$\underline{\text { Introduction: }}$ Multiple previous studies have shown the monoclonal antibody Das-1 (formerly called $7 \mathrm{E}_{12} \mathrm{H}_{12}$ ) specifically recognizes metaplastic and carcinomatous lesions in multiple organs of the gastrointestinal system (e.g. Barrett's esophagus, intestinal-type metaplasia of the stomach, gastric adenocarcinoma, high-grade pancreatic intraepithelial neoplasm, and pancreatic ductal adenocarcinoma) as well as in other organs (bladder and lung carcinomas). Beyond being a useful biomarker in tissue, mAb Das-1 has recently proven to be more accurate than current paradigms for identifying cysts harboring advanced neoplasia. Though this antibody has been used extensively for clinical, basic science, and translational applications for decades, its epitope has remained elusive.

Methods: In this study, we chemically deglycosylated a standard source of antigen, which resulted in near complete loss of the signal as measured by western blot analysis. The epitope recognized by mAb Das-1 was determined by affinity to a comprehensive glycan array and validated by inhibition of a direct ELISA.

Results: The epitope recognized by mAb Das-1 is 3'-Sulfo-Lewis A (3'-Sulfo-Le ${ }^{A}$ ). 3'-Sulfo-Le ${ }^{A}$ is broadly reexpressed across numerous GI epithelia and elsewhere only after metaplastic and carcinomatous transformation.

Discussion: 3'-Sulfo-Le ${ }^{A}$ is a clinically important antigen that can be detected both intracellularly in tissue using immunohistochemistry and extracellularly in cyst fluid and serum by ELISA. The results open new avenues for tumorigenic risk stratification of various gastrointestinal lesions. 


\section{INTRODUCTION}

The monoclonal antibody Das-1 has been used extensively to study metaplasia and cancer in numerous tissues over the last 30 years (Fig. 1, Table 1).(1-17) Das-1 shows broad reactivity in human fetal tissue;(18) however, in adults at homeostasis, expression is primarily restricted to biliary and colonic epithelium as well as skin.(19) Despite, the absence of reactivity in normal healthy tissues of the $\mathrm{Gl}$ foregut, the epitope is reexpressed when these tissues undergo metaplasia that increases risk for cancer and when carcinomatous transformation occurs.(1-17) Thus, the epitope recognized by Das-1 fulfills the criteria for being a true oncofetal antigen. In addition to expression in human tissues, we have recently validated the utility of Das-1 in identifying high risk pancreatic cystic lesions in a large multicenter trial, where we demonstrated that a simple ELISA for Das-1 in cyst fluid outperforms all clinical guidelines in identifying pancreatic cysts harboring malignancy. $(5,6)$

In this study, we aim to identify the oncofetal antigen recognized by mAb Das-1 that has been used as a biomarker for high-risk metaplasia and cancer across numerous tissues in both histology as well as body fluids (serum and pancreatic cyst fluid). Here, using chemical deglycosylation, a comprehensive glycan array, and validation by inhibition of a direct ELISA, we demonstrate that the clinically important epitope of Das-1 is 3'-Sulfo-Le ${ }^{A}$.

\section{METHODS}

For western blots, lyophilized antigen derived from media conditioned by the LS180 colon cancer cell line(5) was deglycosylated using anhydrous trifluoromethanesulfonic acid (TFMS) per manufacturer (Sigma-Aldrich, USA) protocol. Following the deglycosylation reaction, both control and TFMS treated antigen were diluted to the same final volume with Laemelli buffer prior to being applied to the gel. The control reaction was treated in an identical fashion (lyophilization, anisole, and pyrimidine) to the experimental condition; the only difference was TFMS was omitted. 
Both the original Das-1 IgM as well as Das-1 lgG (developed from a hybridoma that had undergone spontaneous isotype switch and thus with identical in vivo reactivity) were assayed against a comprehensive array of 584 glycans provided by the National Center for Functional Glycomics. Briefly, the array was generated from a library of natural and synthetic mammalian glycans with amino linkers printed onto N-hydroxysuccinimide (NHS)-activated glass microscope slides forming covalent amide linkages.(20) The glycan spotting concentration was $100 \mu \mathrm{M} .6$ technical replicates were performed for each antibody. Das- $1 \mathrm{lgM}$ was tested at $5 \mu \mathrm{g} / \mathrm{ml}$ and Das$1 \mathrm{lgG}$ at $5 \mu \mathrm{g} / \mathrm{ml}$ and $50 \mu \mathrm{g} / \mathrm{ml}$. Secondary anti-mouse IgM (488) and anti-Mouse lgG (488) were used at $5 \mu \mathrm{g} / \mathrm{ml}$. The Das-1 IgM and IgG antibodies were provided to CFG and as a fee-for-service and the analysis against the glycan array was completed blinded.

Epitope specificity was confirmed by ELISA of wells coated with antigen $(0.5 \mu \mathrm{g} / \mathrm{well})$, incubated in PBS overnight at $4^{\circ} \mathrm{C}$, blocked with $1 \% \mathrm{BSA}$, and then incubated with $2.5 \mu \mathrm{g}$ of either Das-1 lgG or Das-1 IgM $\pm 200 \mu \mathrm{M}$ of the respective carbohydrate. The reactions were developed after incubation with alkaline phosphate-conjugated anti-mouse $\lg G$ or $\lg M$ and absorption at 405 $\mathrm{nm}$ measured. Separate assays measured effects of competitive inhibition with 0 to $200 \mu \mathrm{M}$ 3'Sulfo-Le ${ }^{A}$.

\section{RESULTS}

Chemical deglycosylation with TFMS of a standard source of concentrated antigen recognized by Das- 1 resulted in near complete loss of Das-1 binding in western blot analysis (93\% and $85 \%$ as measured by $\lg \mathrm{M}$ and $\lg G$, respectively; Fig. 2) indicating the Das-1 epitope depended on glycans. Thus, we determined glycan specificity of the Das-1 IgM and Das-1 IgG antibodies against a comprehensive array of 584 glycans. Both Das-1 IgM and Das-1 IgG preferentially recognized Le $e^{A}$ that had been sulfated at the 3' site of galactose (Fig. 3). The fucose is likely not essential to recognition as both antibodies recognized 3'-Sulfo-Gal $\beta(1-3)$ GlcNAc as well as some 
disulfated glycans, albeit with lower apparent affinity. The antibodies display little-to-no affinity for the non-sulfated, sialylated, or 6'-mono-sulfated counterparts, which are listed as pertinent negatives below the highest ranked hits (Fig. 3). Recognition of the epitope was also independent of net charge, as Mannose-6-Phosphate, another negatively charged sugar, was not recognized by either antibody (Fig. 3). Relative to the IgG, the IgM isotype had similar epitope specificity but exhibited a broader range of measurable affinities (Fig. 3), as might be expected due to the greater avidity of its pentameric quaternary structure.

To confirm the epitope specificity, we performed a direct ELISA using Das-1 against the antigen and found that both Das-1 IgG and IgM were inhibited by 3'-Sulfo-Le ${ }^{A}$, in a dosedependent manner (Fig. 4), and neither the sialylated (3'-Sialyl-Le'; i.e. Ca19-9) nor unsulfated adducts were able to inhibit the reaction. Despite only differing by the Galactose-GlcNAc-fucose arrangement (Fig. 4 Key), Le ${ }^{\mathrm{X}}$ (type II) adducts were not able to competitively inhibit Das-1 binding in the ELISA. Further, the assay was not inhibited by sulfated galactose in the absence of the adjacent GlcNAc and fucose present in Le ${ }^{A}$ (Fig. 4). Thus, both the affinity and inhibitory studies presented here are consistent with 3'-Sulfo-Le ${ }^{A}$ being the epitope recognized by both Das-1 IgG and $\lg \mathrm{M}$.

\section{DISCUSSION}

Aberrant glycosylation patterns (especially acidic modification including sialylation and sulfation) have been identified in numerous types of cancer, and probing for neo-glycosylation epitopes is a common clinical practice used to (1) detect cancer, (2) monitor therapeutic response, and/or (3) evaluate for recurrence. However, typically the utility of these glycosylation epitopes is restricted to a small set of cancers (e.g. CA19-9 for pancreatic cancer). In contrast, 3'-Sulfo-Le ${ }^{A}$ appears to be aberrantly expressed among numerous pre-neoplastic lesions and cancers (Fig. 1, Table 1). 
Sulfation is a posttranslational modification of glycans that adds a negatively charged moiety. Although, these modifications have previously been implicated in numerous cellular functions including cell adhesion and metastatic potential of tumors,(21-25) the ability to specifically detect these antigens in tissue has been stymied by the absence of commercially available lectins or antibodies.(26) Here, we demonstrate that the monoclonal antibodies Das-1 $\lg M$ and $\lg$ specifically recognize 3'-Sulfo-Le ${ }^{\mathrm{A}}$.

The progression from normal tissue to metaplasia to cancer has arguably been best described in the stomach by seminal work of Pelayo Correa and others.(27-30) The pre-cancerous state of chronic atrophic gastritis is characterized by appearance of metaplastic cells deep in the gastric glands.(31) Such Spasmolytic Polypeptide Expressing Metaplasia (SPEM) or pseudopyloric metaplasia cells express Sialyl-Le ${ }^{X}$ antigens that promote binding of the pro-inflammatory, carcinogenic bacteria $H$. pylori. $(32,33)$ Expression of these sialomucins within the columnar epithelial cells is also a defining feature of Type II, incomplete, intestinal-type metaplasia in the stomach.(34) Transition from sialylation to sulfation is the sole feature distinguishing Type II from Type III gastric intestinal metaplasia with the latter being associated with increased risk for progression to cancer.(34) The presence of sulfation in type III GIM has been well correlated with an increased risk of progressing to cancer.(34) Consistent with our data, another group has historically generated an antibody $(91.9 \mathrm{H})$ that recognizes 3 '-Sulfo-Le ${ }^{A}$ and is reactive with Barrett's esophagus(35) and GIM;(36) and phenocopies high iron diamine staining for sulfation in Barrett's and GIM.(36) This antibody recognizes this antigen in the context of a tetra- or pentasaccharide,(37) while here we exclusively used trisaccharides and thus demonstrate that Das-1 recognizes the terminal 3'-Sulfo-Le ${ }^{A}$ trisaccharide and does not require other adjacent sugars.

Uncovering the diagnostically important 3'-Sulfo-Le ${ }^{A}$ modification has important implications. For one, new technologies for specifically detecting this glycan (e.g. mass spectroscopy (38)) may lead to even greater sensitivity in diagnosis of metaplasia and cancer at earlier stages and in a wider variety of fluids and tissues. Moreover, reproducibility of the Das-1 sandwich $\operatorname{ELISA}(5,6)$ 
for clinical laboratory applications may be improved by using pure 3'-Sulfo-Le ${ }^{A}$ as a standard as opposed to the current practice for Das-1: using antigen concentrated from a colon cancer cell line.

It remains to be determined (1) why metaplastic and cancerous tissue of the Gl foregut ubiquitously express this antigen, (2) the necessity of this epitope for metaplastic and oncogenic transformation, (3) what proteins or lipids carry this epitope, and (4) the molecular mechanism by which this epitope is released in pancreatic cyst fluid $(5,6)$ as well as in the serum of individuals with cancer. $(38,39)$ If the cellular processes annotated by these 3 '-Sulfo-Le ${ }^{A}$ antigens confer a proliferative or survival advantage to cancer then specifically inhibiting the sulfation reaction may provide a novel therapeutic strategy for these lethal cellular transformations.

\section{ACKNOWLEDGEMENTS}

Jeffrey W. Brown is supported by the Department of Defense, through the PRCRP program under Award No. W81XWH-20-1-0630, NIH T32 DK007130-42, the Digestive Disease Research Core Centers Pilot and Feasibility Grant as part of P30 DK052574, and the American Gastroenterological Association AGA2021-5101. Jason C. Mills is supported by the National Institute of Diabetes and Digestive and Kidney Diseases (R21DK111369, R01DK094989, R01DK105129, R01DK110406), the Alvin J. Siteman Cancer Center-Barnes Jewish Foundation Cancer Frontier Fund, The National Institutes of Health National Cancer Institute (P30 CA09182, R01CA239645, R01CA246208), and the BETRNet (U54CA163060). The National Center for Functional Glycomics Glycan Array resource and much appreciated assistance with the analysis was supported by R24 GM098791, R24 GM137763, and P41 GM103694. V.K. was supported by the Medical Scientist Training Program Training grant T32 GM07200. Development of mAb Das1 was supported in part by research grants NIDDK, R01 DK47673 and R01 DK63618 to KMD.

\section{AUTHOR CONTRIBUTIONS}


JWB, KKD, VK, JCM: Conceived of the project, funded the research, performed experiments, analyzed data, and wrote the manuscript

KMD: Provided essential reagents, funded the research, and edited the manuscript.

\section{STATEMENT OF ETHICAL ASSURANCE}

JWB \& JCM is the guarantor of this work and, as such, had full access to all of the data in the study and takes responsibility for the integrity of the data and the accuracy of the data analysis. 


\section{REFERENCES}

1. Pantuck AJ, Bancila E, Das KM, et al. Adenocarcinoma of the urachus and bladder expresses a unique colonic epithelial epitope: an immunohistochemical study. J Urol 1997;158:1722-7.

2. Das KM, Prasad I, Garla S, et al. Detection of a shared colon epithelial epitope on Barrett epithelium by a novel monoclonal antibody. Ann Intern Med 1994;120:753-6.

3. DeMeester SR, Wickramasinghe KS, Lord RV, et al. Cytokeratin and DAS-1 immunostaining reveal similarities among cardiac mucosa, CIM, and Barrett's esophagus. Am J Gastroenterol 2002;97:2514-23.

4. Deshpande CG, Shah RN, Yeldandi A, et al. Expression of Das-1 in primary lung adenocarcinomas represents reactivation of an oncofetal pulmonary antigen. Pathobiology 2002;70:343-7.

5. Das KK, Xiao H, Geng $\mathrm{X}$, et al. mAb Das-1 is specific for high-risk and malignant intraductal papillary mucinous neoplasm (IPMN). Gut 2014;63:1626-34.

6. Das KK, Geng X, Brown JW, et al. Cross Validation of the Monoclonal Antibody Das-1 in Identification of High-Risk Mucinous Pancreatic Cystic Lesions. Gastroenterology 2019;157:720-730 e2.

7. Mirza ZK, Das KK, Slate J, et al. Gastric intestinal metaplasia as detected by a monoclonal antibody is highly associated with gastric adenocarcinoma. Gut 2003;52:807-12.

8. Piazuelo MB, Haque S, Delgado A, et al. Phenotypic differences between esophageal and gastric intestinal metaplasia. Mod Pathol 2004;17:62-74.

9. Watari J, Moriichi K, Tanabe H, et al. Biomarkers predicting development of metachronous gastric cancer after endoscopic resection: an analysis of molecular pathology of Helicobacter pylori eradication. Int J Cancer 2012;130:2349-58.

10. O'Connell FP, Wang HH, Odze RD. Utility of immunohistochemistry in distinguishing primary adenocarcinomas from metastatic breast carcinomas in the gastrointestinal tract. Arch Pathol Lab Med 2005;129:338-47.

11. Feng XS, Wang YF, Hao SG, et al. Expression of Das-1, Ki67 and sulfuric proteins in gastric cardia adenocarcinoma and intestinal metaplasia lesions. Exp Ther Med 2013;5:1555-1558. 12. Kawanaka M, Watari J, Kamiya N, et al. Effects of Helicobacter pylori eradication on the development of metachronous gastric cancer after endoscopic treatment: analysis of molecular alterations by a randomised controlled trial. Br J Cancer 2016;114:21-9.

13. Das KK, Brown JW, Fernandez Del-Castillo C, et al. mAb Das-1 Identifies Pancreatic Ductal Adenocarcinoma and High-grade Pancreatic Intraepithelial Neoplasia with High Accuracy. Hum Pathol 2021.

14. Pantuck AJ, Murphy DP, Amenta PS, et al. The monoclonal antibody 7E12H12 can differentiate primary adenocarcinoma of the bladder and prostate. Br J Urol 1998;82:426-30.

15. Onuma EK, Amenta PS, Jukkola AF, et al. A phenotypic change of small intestinal epithelium to colonocytes in small intestinal adenomas and adenocarcinomas. Am J Gastroenterol 2001;96:2480-5.

16. Hahn HP, Blount PL, Ayub K, et al. Intestinal differentiation in metaplastic, nongoblet columnar epithelium in the esophagus. Am J Surg Pathol 2009;33:1006-15.

17. Heidarian A, Das KK, Mino-Kenudson M, et al. Cytology adds value to monoclonal antibody Das-1 testing for detection of high-risk pancreatic cysts. J Am Soc Cytopathol 2021. 
18. Badve S, Logdberg L, Sokhi R, et al. An antigen reacting with das-1 monoclonal antibody is ontogenically regulated in diverse organs including liver and indicates sharing of developmental mechanisms among cell lineages. Pathobiology 2000;68:76-86.

19. Das KM, Vecchi M, Sakamaki S. A shared and unique epitope(s) on human colon, skin, and biliary epithelium detected by a monoclonal antibody. Gastroenterology 1990;98:464-9.

20. Blixt O, Head S, Mondala T, et al. Printed covalent glycan array for ligand profiling of diverse glycan binding proteins. Proc Natl Acad Sci U S A 2004;101:17033-8.

21. Yuen CT, Bezouska K, O'Brien J, et al. Sulfated blood group Lewis(a). A superior oligosaccharide ligand for human E-selectin. J Biol Chem 1994;269:1595-8.

22. Yuen CT, Lawson AM, Chai W, et al. Novel sulfated ligands for the cell adhesion molecule E-selectin revealed by the neoglycolipid technology among O-linked oligosaccharides on an ovarian cystadenoma glycoprotein. Biochemistry 1992;31:9126-31.

23. Galustian C, Lubineau A, le Narvor C, et al. L-selectin interactions with novel mono- and multisulfated Lewisx sequences in comparison with the potent ligand 3'-sulfated Lewisa. J Biol Chem 1999;274:18213-7.

24. Galustian C, Childs RA, Stoll M, et al. Synergistic interactions of the two classes of ligand, sialyl-Lewis(a/x) fuco-oligosaccharides and short sulpho-motifs, with the P- and L-selectins: implications for therapeutic inhibitor designs. Immunology 2002;105:350-9.

25. Galustian C, Childs RA, Yuen CT, et al. Valency dependent patterns of binding of human L-selectin toward sialyl and sulfated oligosaccharides of Le(a) and Le(x) types: relevance to antiadhesion therapeutics. Biochemistry 1997;36:5260-6.

26. Tang H, Partyka K, Hsueh P, et al. Glycans related to the CA19-9 antigen are elevated in distinct subsets of pancreatic cancers and improve diagnostic accuracy over CA19-9. Cell Mol Gastroenterol Hepatol 2016;2:201-221 e15.

27. Saenz JB, Mills JC. Acid and the basis for cellular plasticity and reprogramming in gastric repair and cancer. Nat Rev Gastroenterol Hepatol 2018;15:257-273.

28. Correa P. Human gastric carcinogenesis: a multistep and multifactorial process--First American Cancer Society Award Lecture on Cancer Epidemiology and Prevention. Cancer Res 1992;52:6735-40.

29. de Vries AC, van Grieken NC, Looman CW, et al. Gastric cancer risk in patients with premalignant gastric lesions: a nationwide cohort study in the Netherlands. Gastroenterology 2008;134:945-52.

30. Ota H, Katsuyama T, Nakajima S, et al. Intestinal metaplasia with adherent Helicobacter pylori: a hybrid epithelium with both gastric and intestinal features. Hum Pathol 1998;29:846-50.

31. Goldenring JR. Pyloric metaplasia, pseudopyloric metaplasia, ulcer-associated cell lineage and spasmolytic polypeptide-expressing metaplasia: reparative lineages in the gastrointestinal mucosa. J Pathol 2018;245:132-137.

32. Saenz JB, Vargas N, Mills JC. Tropism for Spasmolytic Polypeptide-Expressing Metaplasia Allows Helicobacter pylori to Expand Its Intragastric Niche. Gastroenterology 2019;156:160-174 e7.

33. Teal E, Dua-Awereh M, Hirshorn ST, et al. Role of metaplasia during gastric regeneration. Am J Physiol Cell Physiol 2020;319:C947-C954.

34. Shah SC, Gawron AJ, Mustafa RA, et al. Histologic Subtyping of Gastric Intestinal Metaplasia: Overview and Considerations for Clinical Practice. Gastroenterology 2020;158:745750. 
35. Azuma N, Endo T, Arimura Y, et al. Prevalence of Barrett's esophagus and expression of mucin antigens detected by a panel of monoclonal antibodies in Barrett's esophagus and esophageal adenocarcinoma in Japan. J Gastroenterol 2000;35:583-92.

36. Bodger K, Campbell F, Rhodes JM. Detection of sulfated glycoproteins in intestinal metaplasia: a comparison of traditional mucin staining with immunohistochemistry for the sulfoLewis(a) carbohydrate epitope. J Clin Pathol 2003;56:703-8.

37. Loveless RW, Yuen CT, Tsuiji H, et al. Monoclonal antibody 91.9H raised against sulfated mucins is specific for the 3'-sulfated Lewisa tetrasaccharide sequence. Glycobiology 1998;8:123742.

38. Tanaka-Okamoto M, Mukai M, Takahashi H, et al. Various sulfated carbohydrate tumor marker candidates identified by focused glycomic analyses. Glycobiology 2017;27:400-415.

39. Zheng J, Bao WQ, Sheng WQ, et al. Serum 3'-sulfo-Lea indication of gastric cancer metastasis. Clin Chim Acta 2009;405:119-26. 


\section{FIGURE LEGENDS}

Figure 1. Unlike normal tissue, metaplastically and oncogenically transformed foregut tissues become reactive to mAb Das-1. Immunohistochemistry of A. Barrett's Esophagus, B. Intestinal Metaplasia of the Stomach, C. Gastric Cancer, D. Pancreatic Ductal Adenocarcinoma. Scale bars presented in bottom left corner of each panel. Bracket labeled "NL" to highlight the absence of staining in normal stomach; contrast with incomplete intestinal-type metaplasia, which expresses 3'-Sulfo-Le ${ }^{A}$. Insets show higher-magnification of boxed areas. White arrowhead: Das1 staining at a cell apex; yellow arrowhead: $3^{\prime}-$ Sulfo-Le $^{A}$ that has been secreted into the extracellular space.

Figure 2. Das-1 IgG and IgM recognize a glycosylation epitope. Chemical deglycosylation of the antigen results in near complete loss of signal as measured by western blot analysis using (A) Das-1 IgM and (B) Das-1 lgG. Quantification of band intensity is presented below each band.

Figure 3. The results of the glycan arrays. Das-1 $\operatorname{lgg}($ at $5 \& 50 \mu \mathrm{g} / \mathrm{mL}$ ) and Das-1 $\operatorname{lgM}$ (at 5 $\mu \mathrm{g} / \mathrm{mL})$ are plotted both linearly $(A, B, C)$ and logarithmically $(D, E, F)$ as the average relative fluorescence units (of 6 technical replicates) plus/minus standard deviation. The top 20 glycans for each arrays are listed and compared to non-sulfated, sialylated, 6'-monosulfated derivatives, as well as other relevant glycans. Colored arrows emphasize that Das-1 IgG and Das-1 IgM recognize the same set of glycans. The complete data sets are provided in supplemental figure 1 (5 $\mu \mathrm{g} / \mathrm{mL} \operatorname{lgG})$, supplemental figure $2(50 \mu \mathrm{g} / \mathrm{mL} \operatorname{lgG})$, and supplemental figure $3(5 \mu \mathrm{g} / \mathrm{mL} \operatorname{lgM})$ and are available for download on the Consortium for Functional Glycomics website (www.functionalglycomics.org). 
Figure 4. Das-1 IgG and IgM specifically recognize 3'-Sulfo-Le'. Direct ELISA using Das-1 $\lg (\mathbf{A})$ or Das-1 $\lg M(B)$ in the absence or presence of several free glycans in solution at 200 $\mu \mathrm{M}$. Direct ELISA using Das-1 IgG (C) or Das-1 IgM (D) against a titration series of 3'-Sulfo-Le ${ }^{\mathrm{A}}$. Data reported as average \pm standard deviation of three technical replicates normalized to the reaction without glycans (PBS). Key: Schematic diagram of the relevant Lewis antigens is provided for reference.

Table 1. Summary of the prior literature describing Das-1 reactivity after metaplasic and/or oncogenic transformation of adult tissues that do not natively express this antigen at homeostasis.

Supplemental Dataset 1. Complete glycan array dataset of Das-1 IgG (5 ug/uL) against the CFG glycan Array. This dataset will also be released for public download at www.functionalglycomics.org.

Supplemental Dataset 2. Complete glycan array dataset of Das-1 lgG (50 ug/uL) against the CFG glycan Array. This dataset will also be released for public download at www.functionalglycomics.org.

Supplemental Dataset 3. Complete glycan array dataset of Das-1 IgM (5 ug/uL) against the CFG glycan Array. This dataset will also be released for public download at www.functionalglycomics.org. 


\section{CHEMICALS/REAGENTS}

Reagents:

D-galactose

D-galactose-3-sulfate potassium salt

D-galactose-4-sulfate sodium salt

D-galactose-6-sulphate

Lewis- $X$ trisaccharide

3'-Sialyl Lewis X tetrasaccharide

Lewis X trisaccharide 3'-sulfate

Lewis-A trisaccharide

3'-Sialyl-Lewis-A tetrasaccharide

3'-Sulfated Lewis A

Antibodies:

Das-1 IgM

Das-1 IgG

Anti-Ms lgG AP conjugated

Anti-Ms IgM AP conjugated

Kits/Consumables:

Immunlon 4 HBX ELISA plates

Phosphatase Substrate

GlycoprofileIV Chemical Deglycosylation Kit Sigma
Sigma

TRC Canada

Sigma

Dextra

Sigma

Biosynth

EMD Millipore

Sigma

Sigma

Biosynth

Leinco

Leinco

Sigma

Sigma

Thermo Scientific

Sigma
Catalog \#G0625

Catalog \#G155295

Catalog \#90572

Catalog \#HGDX253-022

Catalog \#51912

Catalog \#OS04058

Catalog \#434630

Catalog \#44367

Catalog \#S2279

Catalog \#OS05428

Our Hybridoma

Our Hybridoma

Catalog \#A1418

Catalog \#A9688

Catalog \#3855

Catalog \#S0942

Catalog \#PP0510 


\begin{tabular}{|c|c|c|}
\hline Tissue & Transformation & Reference \\
\hline \multirow[t]{2}{*}{ Bladder } & Cancer & Pantuck et al., J Urol 1997; 158: 1722-7. PMID: 9334587 \\
\hline & & Pantuck et al., Br J Urol 1998; 82: 426-30. PMID: 9772883 \\
\hline \multirow[t]{3}{*}{ Esophagus } & Barrett's & Das et al., Ann Intern Med 1994; 120(9): 753-6. PMID: 7511878 \\
\hline & & DeMeester et al., Am J Gastroenterol 2002; 97(10): 2514-23. PMID: 12385432 \\
\hline & & Hahn et al., Am J Surg Pathol 2009; 33(7): 1006-15. PMID: 19363439 \\
\hline Lung & Cancer & Deshpande et al., Pathobiology 2002; 70(6): 343-7. PMID: 12865630 \\
\hline \multirow[t]{4}{*}{ Pancreas } & Cancer & Das et al., Gut 2014; 63(10): 1626-34. PMID: 24277729 \\
\hline & & Das et al., Gastroenterology 2019; 157(3): 720-30. PMID: 31175863 \\
\hline & & Das et al., Hum Pathol. Accepted, in press. PMID: 33524436 \\
\hline & & Heidarian et al., J Am Soc Cytopathol . Accepted, in press . PMID: 33541830 \\
\hline Small Bowel & Adenoma & Onuma et al., Am J Gastroenterol 2001; 96(8): 2480-5. PMID: 11513194 \\
\hline \multirow[t]{5}{*}{ Stomach } & Intestinal-Type Metaplasia & Glickman et al., Am J Surg Pathol 2001; 25(1): 87-94. PMID: 11145256 \\
\hline & & DeMeester et al., Am J Gastroenterol 2002; 97(10): 2514-23. PMID: 12385432 \\
\hline & & Mirza et al., Gut 2003; 52(6): 807-12. PMID: 12740335 \\
\hline & & Piazuelo et al., Mod Pathol 2004; 17(1): 62-74. PMID: 14631367 \\
\hline & & Watari et al., Int J Cancer 2012; 130(10): 2349-58. PMID: 21732341 \\
\hline \multirow[t]{5}{*}{ Stomach } & Cancer & Mirza et al., Gut 2003; 52(6): 807-12. PMID: 12740335 \\
\hline & & O'Connell et al., Arch Pathol Lab Med 2005; 129(3): 338-47. PMID: 15737028 \\
\hline & & Feng et al., Exp Ther Med 2013; 5(6): 1555-8. PMID: 23837030 \\
\hline & & Kawanaka et al., Br J Cancer 2016; 114(1): 21-9. PMID: 26671747 \\
\hline & & Watari et al., Int J Cancer 2012; 130(10): 2349-58. PMID: 21732341 \\
\hline
\end{tabular}


A.

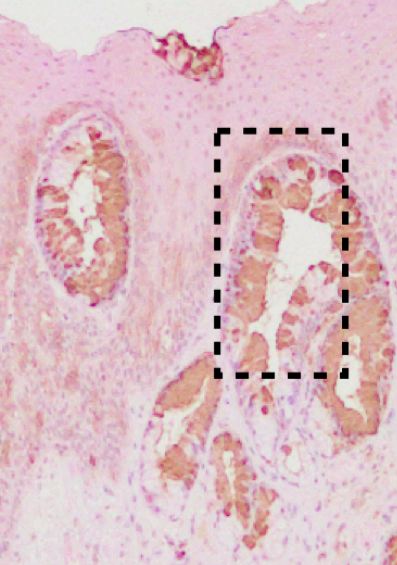

$200 \mu \mathrm{m}$

C.
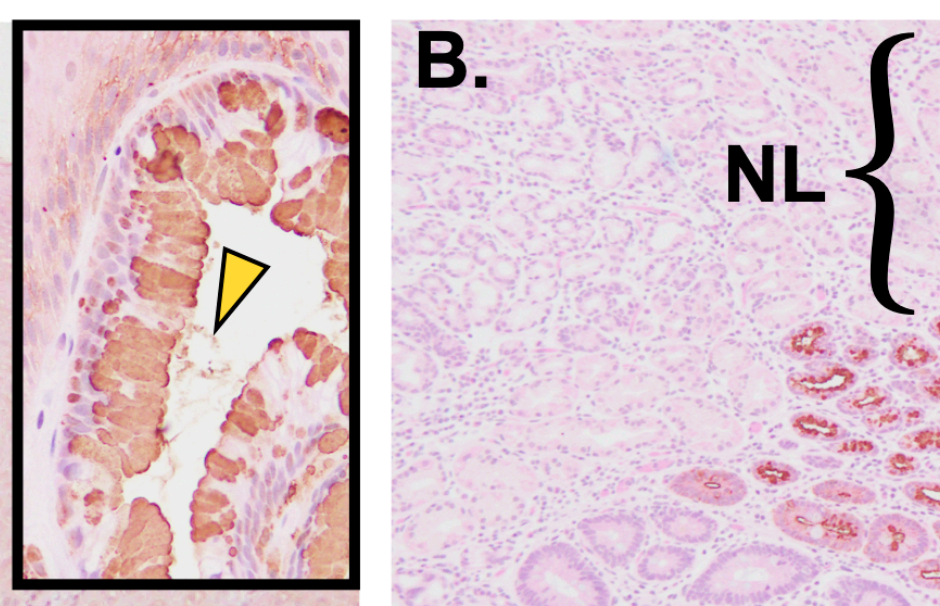

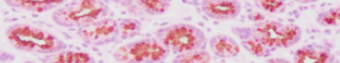

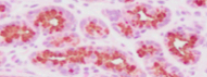

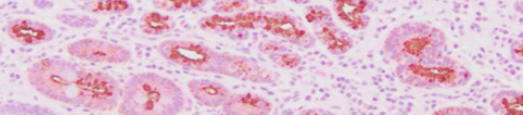

B

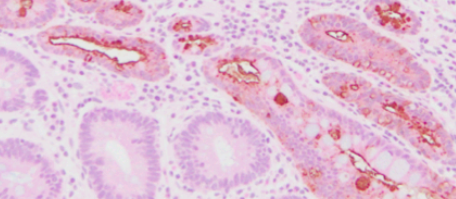

\section{$200 \mu \mathrm{m}$}
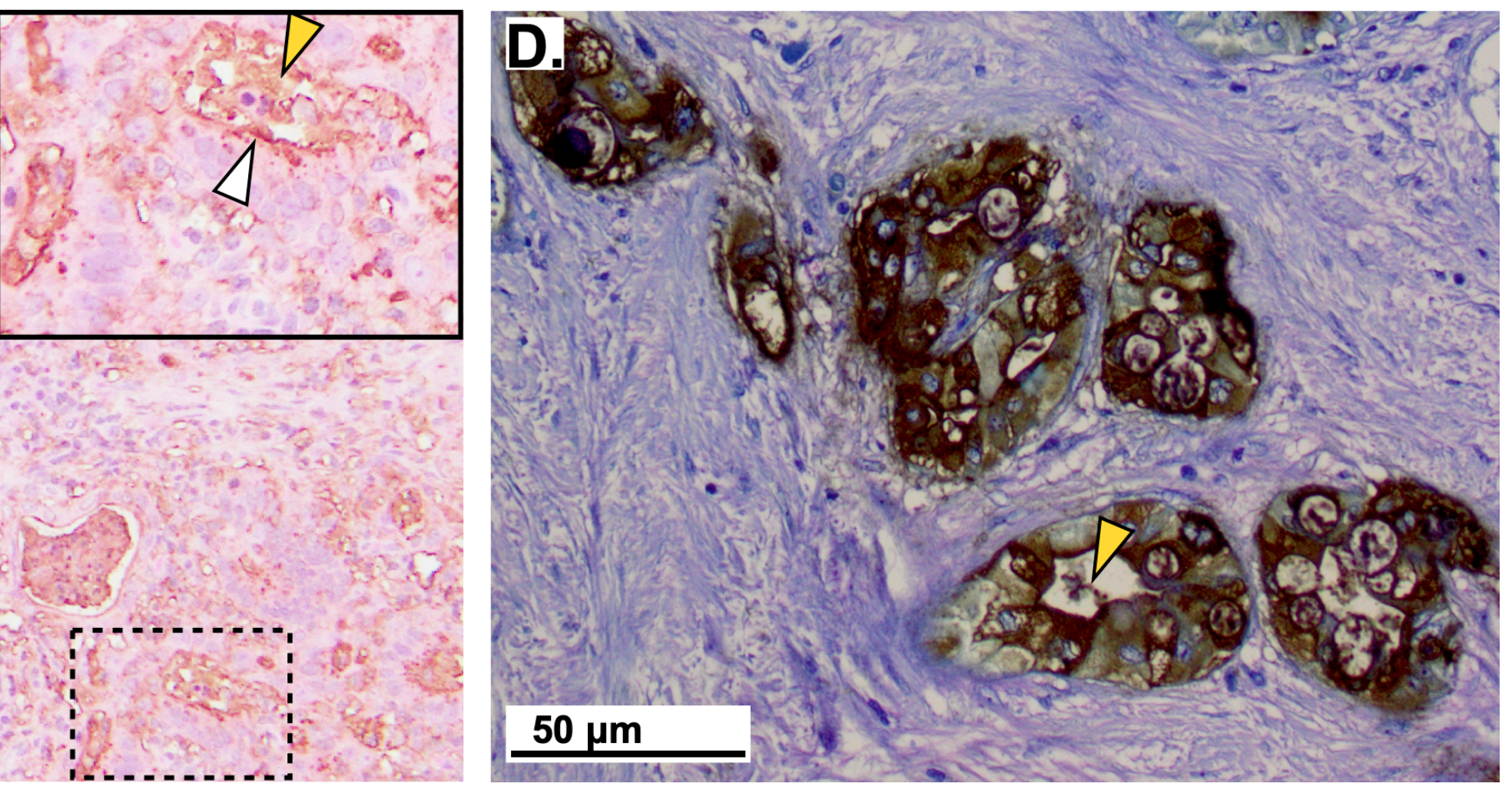
A. $\quad$ B.

TFMS

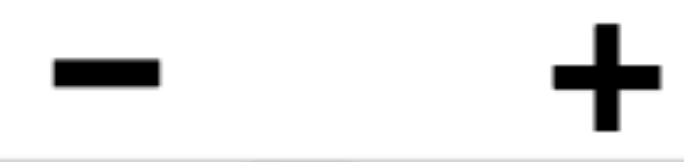

17100011300

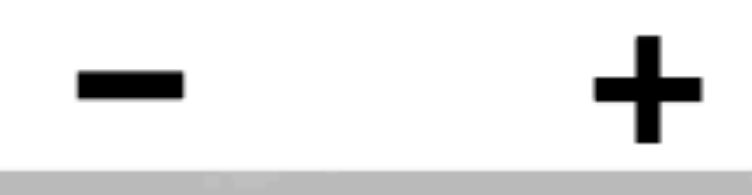

123001910

$250 \mathrm{KDa}$

148 KDa

$98 \mathrm{KDa}$

$64 \mathrm{KDa}$

$50 \mathrm{KDa}$

$36 \mathrm{KDa}$

22 KDa

$16 \mathrm{KDa}$

$6 \mathrm{KDa} \rightleftharpoons$ Das-1 IgM
$4 \mathrm{KDa}$

Das-1 IgG 

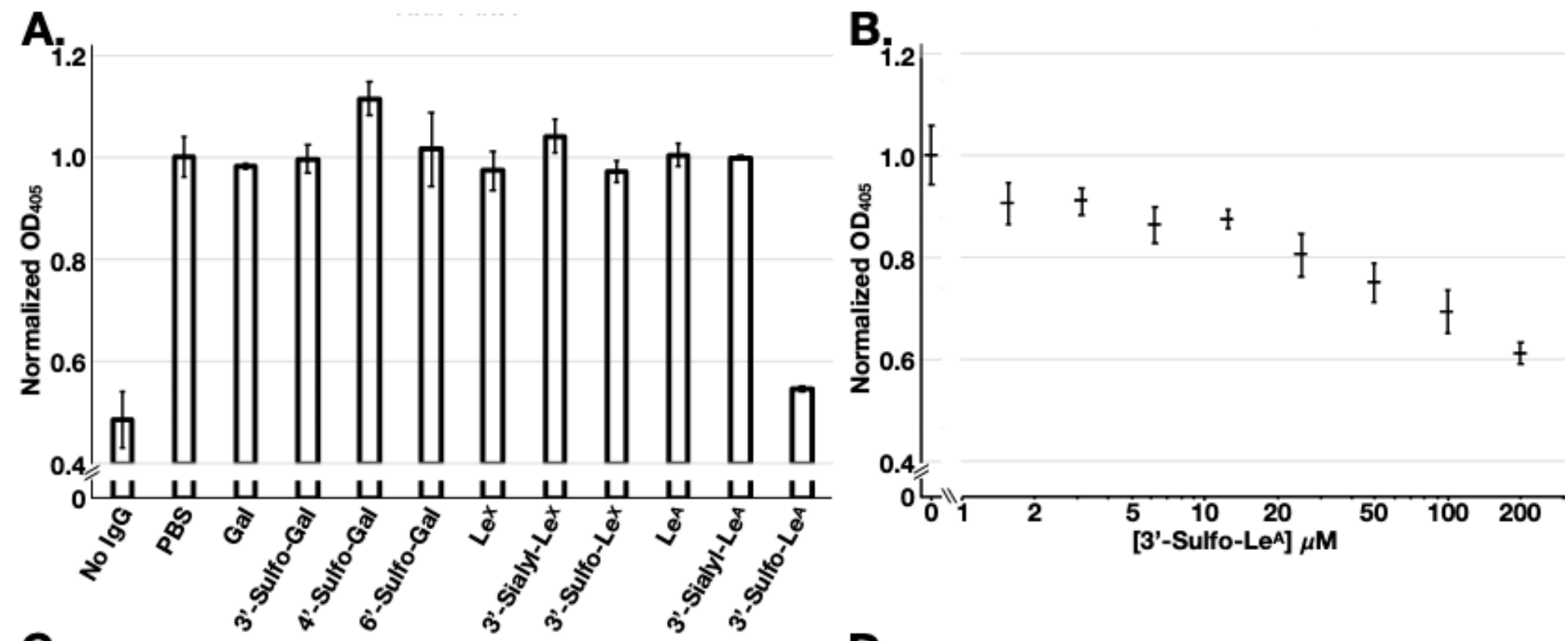

C.

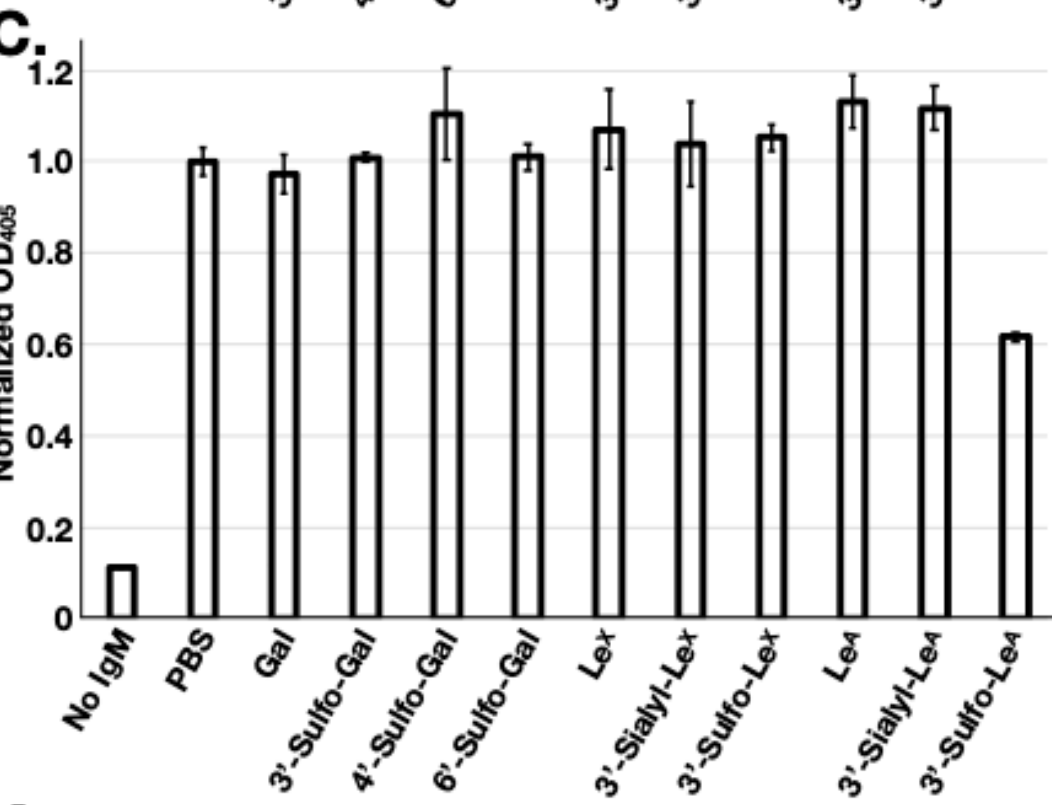

$\mathbf{D}_{\mathbf{i} .2}$

Key: Type 1 LacNAc (Gal $\beta 1,3$ GICNAc)

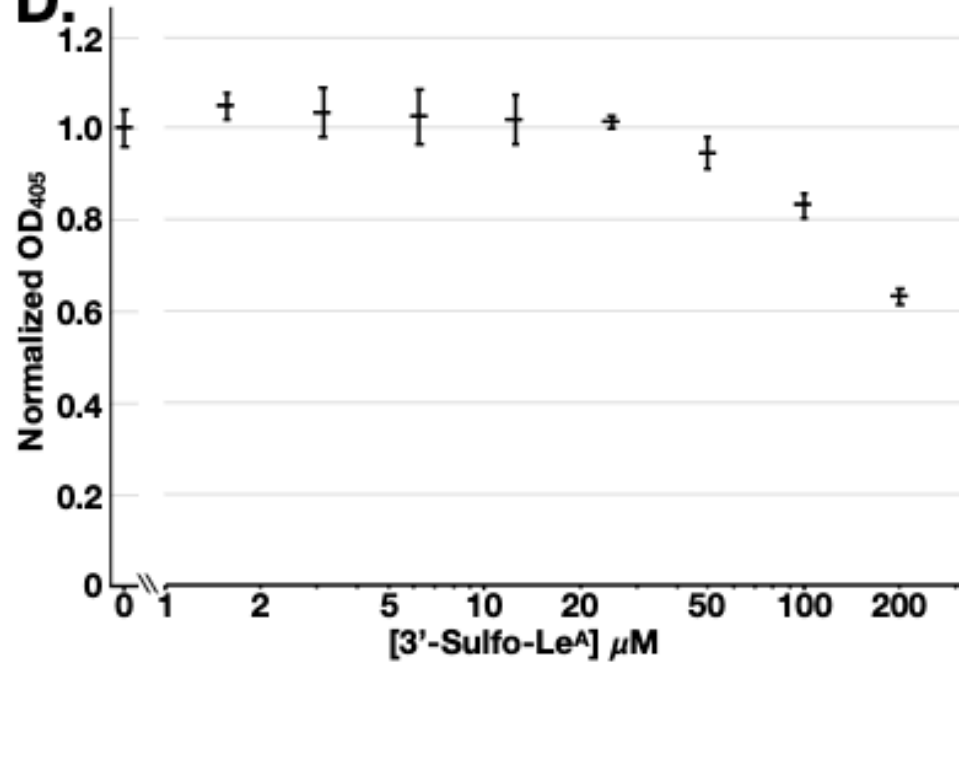

Type 2 LacNAc (Gal $\beta$ 1,4 GIcNAc)

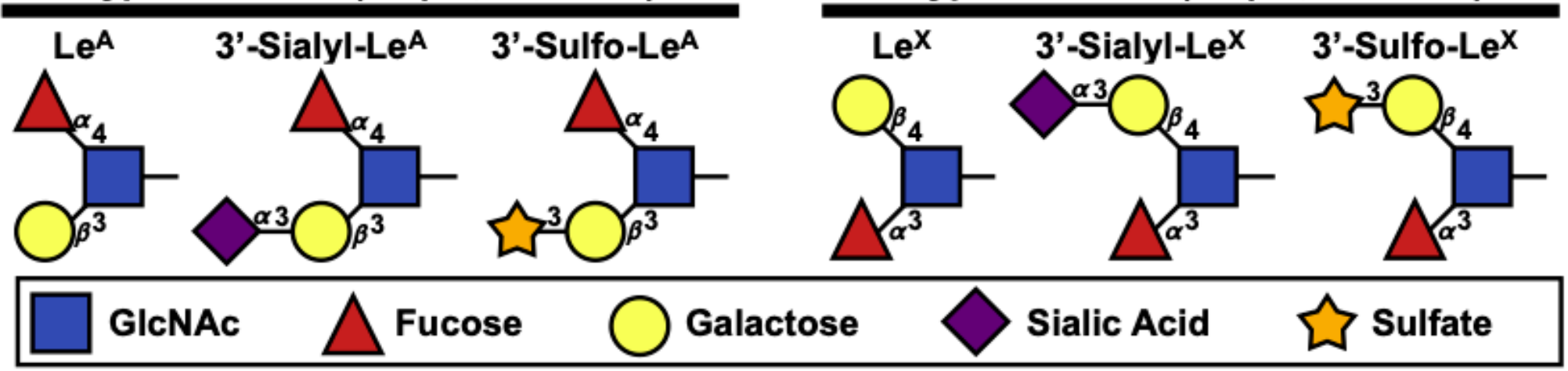

